\title{
Cardiovascular Parameters in Rat Model of Chronic Renal Failure Induced by Subtotal Nephrectomy
}

\author{
J. ŠVÍGLEROVÁ ${ }^{1}$, J. KUNCOVÁ ${ }^{1}$, L. NALOS ${ }^{1}$, Z. TONAR ${ }^{2}$, D. RAJDL ${ }^{3}$, M. S̆TENGL $^{1}$ \\ ${ }^{1}$ Department of Physiology, Faculty of Medicine in Plzeň, Charles University in Prague, Czech \\ Republic, ${ }^{2}$ Department of Histology and Embryology, Faculty of Medicine in Plzeň, Charles \\ University in Prague, Czech Republic, ${ }^{3}$ Institute of Clinical Biochemistry and Hematology, Faculty \\ of Medicine and Teaching Hospital in Plzeň, Charles University in Prague, Czech Republic
}

Received February 2, 2010

Accepted March 26, 2010

\section{Summary}

Chronic renal failure (CRF) is associated with high incidence of cardiovascular complications. To clarify pathogenesis of CRF numerous animal models have been developed. The aim of our work was to describe methodology of subtotal surgical renal ablation in rat and to characterize some biochemical and cardiovascular parameters of this animal model. Male rats underwent 5/6 surgical nephrectomy or sham operations in two steps. The following parameters were measured on day 10 and in week 10 after the surgery: plasma concentrations of creatinine and urea, blood pressure, resting heart rate, chronotropic response to atropine and metipranol, heart ventricles weight, contraction parameters and action potential duration in the left ventricle. Increased serum concentrations of creatinine and urea, decreased creatinine clearance, polyuria and alteration of the remnant kidney tissue were found in CRF rats. Changes in cardiovascular parameters identified after subtotal nephrectomy resembled alterations of cardiovascular system in uremic patients and included hypertension, elevated resting heart rate, diminished parasympathetic cardiac tone, hypertrophy of the left ventricle associated with weakened force of contraction, prolonged contraction and relaxation and shortening of action potential duration. These data suggest that the present model can be a useful tool in the study of CRF and its cardiovascular complications.

\section{Key words}

Rat • Renal failure - Subtotal nephrectomy - Cardiovascular complications

\section{Corresponding author}

J. Švíglerová, Department of Physiology, Faculty of Medicine, Charles University, Lidická 1, 30100 Plzeň, Czech Republic. Fax: +420 377593349. E-mail: jitka.sviglerova@lfp.cuni.cz

\section{Introduction}

Chronic renal failure (CRF) is the end-stage of most chronic renal diseases. The incidence of cardiovascular complications including hypertension, left ventricle hypertrophy, both systolic and diastolic dysfunctions, autonomic neuropathy and arrhythmia in CRF is in average about 20 times higher compared to normal population. The important fact is that the risk of cardiovascular complications in younger patients is even 100 times higher. Cardiovascular diseases (mainly heart failure, ischemic heart disease or sudden cardiac death) are the leading causes of death in CRF patients and account for approximately $50 \%$ of all deaths in CRF population (Tesař 2006). Although the cardiovascular complications of CRF are intensely investigated the pathogenesis of some of them has not yet been fully clarified.

In experimental animal studies, CRF is the most often induced by subtotal nephrectomy (remnant kidney model). This model may be achieved by either ligation of renal vessels supplying the renal poles or surgical removal of both renal poles followed by contralateral nephrectomy (Chow et al. 2003). The technical difficulties are obvious in both approaches: the ligation method gives heterogeneous results due to anatomical 
variations of the renal artery among different rats (Liu et al. 2003) and the surgical one presents the risk of haemorrhage and higher mortality due to surgical complications (Boudet et al. 1978). Another way to produce experimental renal failure is the administration of nephrotoxic agents. Antibiotic adriamycin (Okuda et al. 1986), cisplatin (Heidemann et al. 1990), uranyl nitrate (Fukuda and Kopple 1980) or aristolochic acid (Debelle et al. 2004) have been evaluated for inducing renal damage. The procedure of chemical nephrectomy is relatively easy but the cardiovascular system-related disadvantages of pharmacological models predominate: nephrotoxic drugs exert a lot of undesirable side-effects inclusive cardiotoxicity. Moreover, some of them modify the development of renal and subsequent cardiac lesions (Chow et al. 2003). The aim of our work was to describe the methodology of surgical subtotal nephrectomy in rat, to characterize basic features of this model including the progressive changes in cardiovascular functions and to assess the similarity of this animal model with the pattern of CRF in humans.

\section{Methods}

All experiments were carried out in accordance with European Directive for the Protection of Vertebrate Animals Used for Experimental and Other Scientific Purposes (86/609/EU), the relevant Guidelines of the Czech Ministry of Agriculture for scientific experimentation on animals and were approved by the University Committee for Experiments on Laboratory Animals (Charles University, Czech Republic).

\section{Rat model of chronic renal failure}

Four-months-old Wistar male rats (Velaz, Prague, Czech Republic) were randomly allocated into subtotal nephrectomy (CRF, $\mathrm{n}=60)$ and sham-operation $(n=20)$ groups. All operations were performed under total anesthesia by intraperitoneal injection of sodium pentobarbital $(100 \mathrm{mg} / \mathrm{kg}$, i.p., Sigma Aldrich, Czech Republic). In CRF group the left kidney was exposed through a lateral dorsal incision and decapsulated. The renal vessels were clamped and both poles $(2 / 3$ of the functional kidney mass) were resected. The cut surface was cauterized and then treated by hemostat Lyostypt (B.Braun, Germany). Eventually, the vessel clamp was removed and the renal stump was returned into the abdominal cavity. Two weeks later the right renal vessels were ligated and the total right nephrectomy through lateral dorsal incision was done to achieve $5 / 6$ reduction of the total renal mass. The sham operation in control rats consisted of renal evacuation, decapsulation and returning the intact kidney into the abdominal cavity performed bilaterally in a two-week interval. The strict aseptic rules were respected and the special care was taken to prevent damage to the adrenals during operations. The incisions were sutured in two layers and covered by a liquid bandage. After each operation, rats were treated by onetime application of antibiotic marbofloxacinum $(5 \mathrm{mg} / \mathrm{kg}$, s.c., Marbocyl, Vétoquinol, Czech Republic) and housed individually with free access to food and water.

Body weight was measured weekly after the second surgery. Creatinine concentration in serum and urine and urea concentration in serum were measured on a routine autoanalyzer system (Olympus AU 640, Mishima, Japan) before, 10 days and 10 weeks after sham-operation or subtotal nephrectomy. Blood samples were taken from the tail vein. At the same points of time the animals were placed into metabolic cages and 24-hours samples of urine were collected. Glomerular filtration rate was determined by means of creatinine clearance.

To evaluate the morphological status of the kidney, the animals were sacrificed, the kidney wet weight was determined and histological analysis of renal tissue was performed at week 10 after sham operation or subtotal nephrectomy. The kidneys were processed by common paraffin technique and cut perpendicularly to the kidney surface into series of 200 slices with thickness of $5 \mu \mathrm{m}$. The sections were stained with hematoxylin-eosin and Verhoeff's hematoxylin with green trichrome, the latter providing a sufficient contrast between the wall of the nephron and the interstitial connective tissue.

Animals were used for experiments 10 days and 10 weeks after subtotal nephrectomy or sham operation, if not stated otherwise.

\section{Measurement of blood pressure and heart rate}

Blood pressure and heart rate were recorded in conscious animals before, 10 days and 10 weeks after operations. The values of systolic and diastolic pressures were measured by the tail-cuff method using an RTBP apparatus (Kent Scientific Co., USA). To register heart rate the rats were placed in a small chamber with floor electrodes connected to an electrocardiograph (EKG Seiva Praktik, Czech Republic). The tonic influence of parasympathetic innervation on the heart rate was studied using muscarinic receptor antagonist atropine $(4 \mathrm{mg} / \mathrm{kg}$ of 
body weight, s.c., atropine sulphate, Hoechst-Biotika, Slovac Republic) applied after pre-treatment with beta adrenergic receptor blocker metipranolol (2 mg/kg, s.c., Sigma-Aldrich, Czech Republic). The tonic influence of sympathetic innervation was estimated by a reversed application of the above mentioned blockers. The changes in heart rate were calculated as the differences between values recorded before and 20 minutes after the drug administration.

\section{Contraction and electrophysiology experiments}

Animals were anaesthetized with intraperitoneal injection of urethane $(1.5 \mathrm{~g} / \mathrm{kg}$ body weight) 10 minutes after having received heparin (500 U, i.p.) and their hearts were quickly excised. The whole heart, the left ventricle including septum and the free right ventricular wall were weighed. Then the papillary muscles were dissected from the left ventricle and placed in an experimental chamber and attached to an isometric force transducer F30 (Hugo Sachs, Germany). The resting tension was set to reach 90-95\% of maximum developed force at stimulation frequency of $1 \mathrm{~Hz}$. Double-chamber stimulation (stimulator Pulsemaster A300, WPI, USA) was used. The square-wave voltage pulses had duration of $1 \mathrm{~ms}$ and amplitude $\geq 50 \%$ above threshold. The preparation was perfused with $36{ }^{\circ} \mathrm{C}$, oxygenated Tyrode solution at a constant flow rate $(6-10 \mathrm{ml} / \mathrm{min})$. After a stabilization period ( $>30$ minutes) the steady-state contractions and action potentials at stimulation frequencies $0.5,1,2$ and $3 \mathrm{~Hz}$ were recorded. Time course of contraction-relaxation cycle was characterized using time-to-peak (time from resting tension to the peak of contraction, TTP), time-to-50\% relaxation $\left(\mathrm{R}_{50}\right)$ and time-to- $90 \%$ relaxation $\left(\mathrm{R}_{90}\right)$. The resting tension was taken as zero. The membrane potential was measured with glass microelecrodes (filled with $3 \mathrm{~mol} / \mathrm{l} \mathrm{KCl}$, resistance $>20 \mathrm{M} \Omega$ ). Action potential duration was measured at $50 \%$ and $90 \%$ levels of repolarization $\left(\mathrm{APD}_{50}, \mathrm{APD}_{90}\right)$. Data were recorded and analyzed using the data acquisition system DiSys (Merlin, Czech Republic).

\section{Solutions and chemicals}

The composition of the Tyrode solution was following (in mmol/l): $\mathrm{NaCl} 137, \mathrm{KCl} 4.5, \mathrm{MgCl}_{2} 1$, $\mathrm{CaCl}_{2} 2$, glucose 10, Hepes 5; pH was adjusted to 7.4 with $\mathrm{NaOH}$. Chemicals were from Sigma Aldrich or Lachema (Czech Republic). All chemicals were of analytical grade.

\section{Data analysis}

Results are presented as means \pm S.E.M. Statistical differences were tested by unpaired two-tailed Student's t-test or by analysis of variance (ANOVA) followed by post hoc Fisher's Least Significant Difference test. Normality of populations and homogeneity of variances were tested before each ANOVA. The results were considered significantly different when $\mathrm{p}<0.05$.

\section{Results}

\section{Rat model of chronic renal failure}

To verify the development of CRF, daily volume of urine, serum creatinine and urea levels were monitored and glomerular filtration rate was calculated before, 10 days and 10 weeks after surgery. There were no significant differences between sham and CRF groups before surgery. Serum creatinine and urea concentrations and daily volume of urine were significantly higher in CRF group 10 days after surgery and they did not significantly change to the end of the experiment. At week 10 of the experiment serum creatinine was threefold and serum urea fourfold higher in CRF rats. Creatinine clearance dropped due to $5 / 6$ nephrectomy to $30 \%$ on day 10 and to $40 \%$ of control value in week 10 of the experiment (Table 1).

Functional markers of chronic renal failure were associated with morphological changes in the remnant kidney. The weight of renal stump (relative to body weight) in nephrectomized rats was significantly higher compared to the relative weight of the intact kidney in control ones, reaching $0.332 \pm 0,029 \%(n=6)$ in sham and $0.557 \pm 0,051 \%(n=6)$ in CRF 10 weeks after operations. Figure 1 documents the abnormal shape and the atypical pale colour of the renal stump in subtotally nephrectomized rats. Histological examination showed compensatory hypertrophy of renal mass mainly of tubular system in CRF rats. Capsular system was depressed in several areas. No necrotic areas were found but the medullary pyramids were distorted (Fig. 1). 
Table 1. Biochemical data from sham-operated (control) and subtotally nephrectomized rats. 0 - values before surgery, 10D - ten days after surgery, 10W - ten weeks after surgery, GFR - glomerular filtration rate measured by clearance of creatinine, * significantly different from control $(p<0.05)$.

\begin{tabular}{lcccccc}
\hline & $\begin{array}{c}\text { sham0 } \\
(\mathbf{n = 2 0})\end{array}$ & $\begin{array}{c}\text { CRFo } \\
\mathbf{( n = 6 0 )}\end{array}$ & $\begin{array}{c}\text { sham10D } \\
\mathbf{( n = 6 )}\end{array}$ & $\begin{array}{c}\text { CFR10D } \\
(\mathbf{n = 8})\end{array}$ & $\begin{array}{c}\text { sham10W } \\
\mathbf{( n = 1 5 )}\end{array}$ & $\begin{array}{c}\text { CRF10W } \\
(\mathbf{n}=\mathbf{1 8})\end{array}$ \\
\hline Serum creatinine $(\mu \mathrm{mol} / \mathrm{l})$ & $49 \pm 0.7$ & $51.7 \pm 1.8$ & $52.5 \pm 1.6$ & $150.6 \pm 10^{*}$ & $52.1 \pm 2.1$ & $146.7 . \pm 9.9 *$ \\
Serum urea $(\mathrm{mmol} / \mathrm{l})$ & $7.0 \pm 0.9$ & $6.6 \pm 1.1$ & $6.5 \pm 0.2$ & $26.9 \pm 2 *$ & $6.7 \pm 0.2$ & $25.6 \pm 3.1^{*}$ \\
Urine volume $(\mathrm{ml} / 24 \mathrm{hod})$ & $15.2 \pm 1.2$ & $14.9 \pm 1.4$ & $16.8 \pm 0.9$ & $49 \pm 3 *$ & $15.67 \pm 1.2$ & $39.44 \pm 3.2 *$ \\
GFR $(\mathrm{ml} / \mathrm{min})$ & $2.3 \pm 0.19$ & $2.4 \pm 0.22$ & $2.1 \pm 0.11$ & $0.62 \pm 0.04 *$ & $2.6 \pm 0.18$ & $0.99 \pm 0.04^{*}$ \\
\hline
\end{tabular}
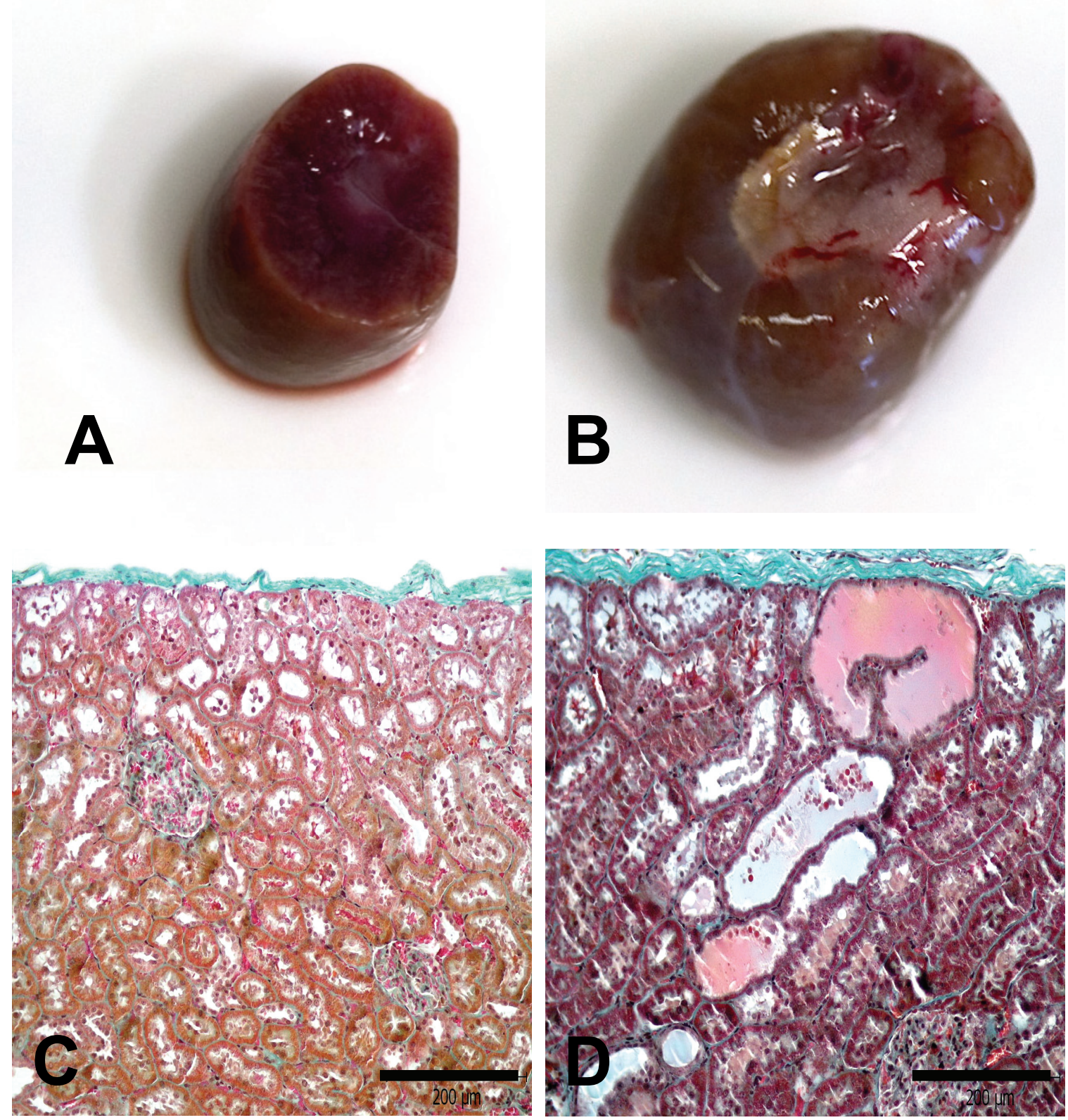

Fig. 1. Macroscopic and microscopic changes in the kidney 10 weeks after subtotal nephrectomy. Upper part: cross section of the intact kidney in sham-operated rat (A) and the renal stump of subtotally nephrectomized rat (B), magnification 2.5 (A, B). Lower part: hematoxylin-eosin stained sections of the intact kidney from sham-operated rat (C) and renal stump from subtotally nephrectomized rat (D). Scale bar indicates $200 \mu \mathrm{m}$ (C, D). 
Table 2. Effect of subtotal nephrectomy on blood pressure and heart rate. 0 - values before surgery, 10D - ten days after surgery, $10 \mathrm{~W}$ - ten weeks after surgery, BPsyst - systolic blood pressure in $\mathrm{mmHg}$, BPdiast - diastolic blood pressure in $\mathrm{mm} \mathrm{Hg}, \mathrm{HR}-\mathrm{resting}$ heart rate, $\mathrm{HR}_{\mathrm{ATR}}$ - increase in heart rate after atropine administration to animals pre-treated by metipranolol, $\mathrm{HR}_{\mathrm{MP}}-$ decrease in heart rate after metipranolol administration to rats pre-treated by atropine, * significantly different from control $(p<0.05)$.

\begin{tabular}{lcccccc}
\hline & $\begin{array}{c}\text { sham0 } \\
(\mathbf{n = 2 0})\end{array}$ & $\begin{array}{c}\text { CRF0 } \\
(\mathbf{n = 6 0})\end{array}$ & $\begin{array}{c}\text { sham10D } \\
\mathbf{( n = 6 )}\end{array}$ & $\begin{array}{c}\text { CRF10D } \\
\mathbf{( n = 8 )}\end{array}$ & $\begin{array}{c}\text { sham10W } \\
(\mathbf{n = 1 5})\end{array}$ & $\begin{array}{c}\text { CRF10W } \\
(\mathbf{n = 1 8})\end{array}$ \\
\hline BPsyst & $135 \pm 7.3$ & $141 \pm 8$ & $136.7 \pm 7.9$ & $178.1 \pm 6.5^{*}$ & $138 \pm 2.2$ & $201.8 \pm 4.1^{*}$ \\
BPdiast & $98.9 \pm 8$ & $97.9 \pm 8$ & $106.9 \pm 5$ & $132.3 \pm 3^{*}$ & $102.2 \pm 2.9$ & $133 \pm 5.1^{*}$ \\
$H R$ & $355 \pm 12$ & $349 \pm 11$ & $340 \pm 9$ & $362 \pm 15$ & $345 \pm 9$ & $399 \pm 11^{*}$ \\
$H R_{A T R}$ & $85 \pm 9$ & $75 \pm 8$ & $75 \pm 10$ & $40 \pm 9^{*}$ & $79 \pm 9$ & $45 \pm 8^{*}$ \\
$H R_{M P}$ & $121 \pm 10$ & $131 \pm 11$ & $124 \pm 11$ & $106 \pm 10$ & $125 \pm 5$ & $118 \pm 9$ \\
\hline
\end{tabular}
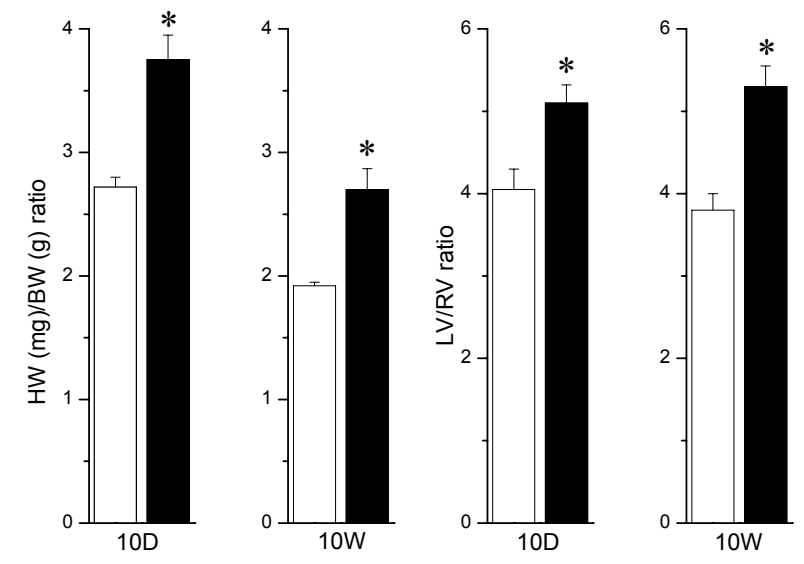

Fig. 2. Relative weight of the heart and the left ventricle to right ventricle weight ratio in control and subtotally nephrectomized rats. Empty bars: sham operated rats $(n=6$ and $n=15,10$ days and 10 weeks after operation, respectively), filled bars: subtotally nephrectomized rats $(n=8$ and $n=18,10$ days and 10 weeks after operation, respectively), HW - weight of the whole heart, BW - body weight, LV - weight of the left ventricle including septum, RV - weight of the free right ventricular wall, 10D - ten days after surgery, $10 \mathrm{~W}-$ ten weeks after surgery, $*$ significantly different from the value in control group $(p<0.05)$.

\section{Blood pressure and heart rate}

The values of both systolic and diastolic pressure were significantly higher in the CRF group 10 days and 10 weeks after $5 / 6$ nephrectomy. The resting heart rate showed rising tendency in subtotally nephrectomized rats but the significant difference was found only at week 10 after surgery. Cardiac parasympathetic tone measured by an increase in heart rate after application of muscarinic blocker atropine to rats pre-treated by beta blocker metipranolol was significantly lower in CRF rats. The sympathetic cardiac tone evaluated like a decrease in heart rate after metipranolol application in the presence of atropine was comparable in both experimental groups (Table 2).
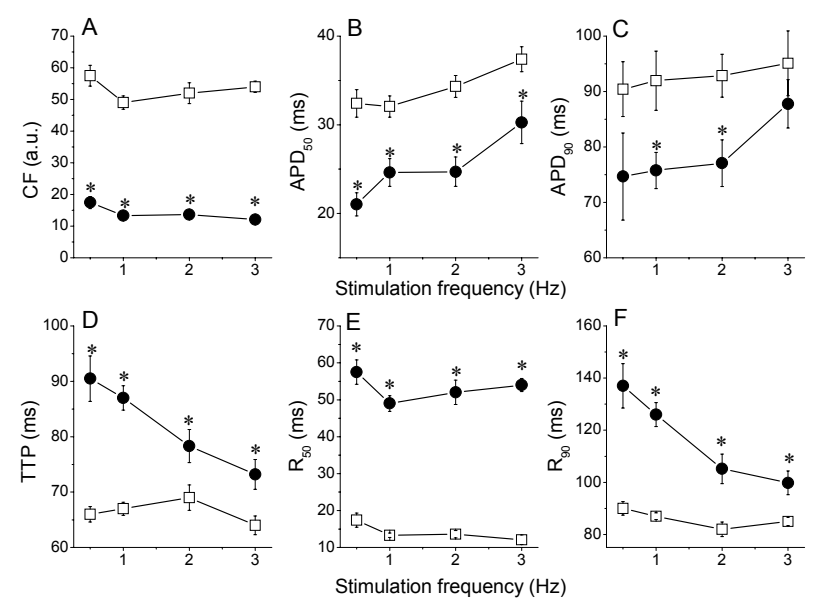

Fig. 3. Contractile force, action potential duration, time to peak and relaxation times in sham and subtotally nephrectomized rats 10 weeks after surgery. A. Contractile force (CF). B. Action potential duration at $50 \%$ repolarization $\left(A P_{50}\right)$. C. Action potential duration at $90 \%$ repolarization $\left(A P D_{90}\right)$. D. Time to peak (TTP). E. Time-to-50 \% relaxation $\left(R_{50}\right)$. F. Time-to- $90 \%$ relaxation $\left(R_{90}\right)$. Open squares: sham rats (control; $\left.n=6\right)$, filled circles: CRF rats $(n=8), *$ significantly different from control $(p<0.05)$.

\section{Contraction and electrophysiology experiments}

Heart weight to body weight ratio of nephrectomized rats was significantly higher in comparison with the sham-operated ones at both day 10 and week 10 of the experiment. Since then the left ventricle (including the septum) to right ventricle weight ratio was significantly increased, the increase in relative heart weight probably resulted from hypertrophy of the left ventricle (Fig. 2).

To study the effect of CRF on the left ventricle functions, contractile force (CF), time to peak (TTP), time-to- $50 \%$ relaxation $\left(\mathrm{R}_{50}\right)$ and time-to- $90 \%$ relaxation $\left(\mathrm{R}_{9_{0}}\right)$ in the left papillary muscle were measured 10 days and 10 weeks after 5/6 nephrectomy or sham operation. Subtotal nephrectomy weakened CF and 
prolonged TTP, $\mathrm{R}_{50}$ and $\mathrm{R}_{90}$ significantly at all stimulation frequencies tested and at both time point (Fig. 3 A, B, C).

The action potential duration measured at $50 \%$ level of repolarization $\left(\mathrm{APD}_{50}\right)$ was significantly shorter in CRF than in sham-operated controls at all stimulation frequencies whereas the action potential duration measured at $90 \%$ level of repolarization $\left(\mathrm{APD}_{90}\right)$ was shortened only at stimulation frequencies 1 and $2 \mathrm{~Hz}$ 10 weeks after surgery (Fig. 3 D, E, F).

\section{Discussion}

The present paper describes the basic features of the rat model of chronic renal failure induced by $5 / 6$ surgical nephrectomy and the impact of this intervention on some cardiovascular parameters in the early (on day 10 after surgery) and the late (in week 10) stages of a kidney disorder. The typical biochemical and morphological signs of renal failure including increased serum concentrations of creatinine and urea, decreased clearance of creatinine, polyuria and the alteration of the remnant kidney tissue were found. The significant changes of cardiovascular parameters identified 10 weeks after subtotal nephrectomy comprised hypertension, elevated resting heart rate, diminished parasympathetic cardiac tone, hypertrophy of the left ventricle associated with weakened contraction force, prolongation of contraction and relaxation and shortening of action potential duration. All the above mentioned alterations of cardiovascular functions except the significant increase in resting heart rate were also observed already 10 days after $\mathrm{CRF}$ induction. In addition to the frequently used rat model of $5 / 6$ nephrectomy we also tested $3 / 4$ or $7 / 8$ nephrectomy models in our pilot experiments. The less radical reduction of the renal mass led to only moderate increase in serum creatinine and urea concentrations immediately after surgery followed by the restoration of biochemical parameters during next two weeks. On the other hand, the reduction to only of $1 / 8$ of the functional renal mass resulted in severe uraemia and high mortality (almost $80 \%$ ) of experimental animals in the first week after 7/8 nephrectomy (Švíglerová, unpublished data).

Hypertension in CRF patients is an important factor of the left ventricular hypertrophy, cardiac failure, coronary artery disease and arrhythmia. The seriousness and incidence of hypertension increases as renal functions deteriorate (Zoccali et al. 2004). Development of hypertension in the rat model is dependent on the technique used. Hypertension is a typical feature in remnant kidney model achieved by unilateral nephrectomy followed by surgical excision with cauterization (used in our work) of $2 / 3$ of the contralateral kidney. Renal reduction produced by surgical ablation without cauterization results in a serious rise of blood pressure only if the intervention is performed at a younger age (Griffin et al. 1994). Similarly to humans, the severity of hypertension in the rat model is inversely proportional to the amount of the residual functional renal mass (Brenner and Anderson 1992).

The cardiac autonomic neuropathy plays a key role in pathogenesis of blood pressure alterations (both hypertension and hypotension), arrhythmia and sudden cardiac death in patients with CRF. Despite the fact that uremic autonomic neuropathy is intensely studied by means of many non-invasive and invasive tests the mechanism of this malfunction remains partly unclear (Lugon et al. 2003). The diverse results of various diagnostic methods studying autonomic neuropathy are explained by their different sensitivity (Sahin et al. 2006, Savica et al. 2001). The clinical picture of cardiac autonomic neuropathy is also influenced by antihypertensive drugs that must be often administered to uremic patients. In our experiments, resting heart rate was significantly higher in CRF rats. To assess the involvement of parasympathetic and sympathetic nervous systems in the proved tachycardia, changes of heart rate after the administration of muscarinic blocker atropine and beta adrenergic blocker metipranolol were measured. The decreased cardiac parasympathetic tone was found in subtotally nephrectomized rats whereas the sympathetic tone was not markedly changed. Our results correspond with findings in a number of studies in which the alteration of parasympathetic branch of autonomic nervous system rather than impairment of sympathetic one contributes to autonomic neuropathy in patients with CRF (Zoccali et al. 1982, Vita et al. 1990, Giordano et al. 2001).

The association of chronic renal failure with myocardial contractile dysfunction is generally known but the contribution of systolic and diastolic dysfunctions to heart disease in CRF patients remains a matter of debate (Burke and Solomon 2000, Wanic-Kossowska et al. 2003). The recent studies suggest that the left ventricular hypertrophy commonly present in CRF results in either systolic or diastolic dysfunction and finally leads to cardiac failure (Zoccali et al. 2004). We observed that chronic renal failure induced by $5 / 6$ surgical nephrectomy 
diminished contraction force and prolonged both contraction and relaxation time in the left ventricle papillary muscle. The described changes caused probably by remodelling of uremic myocardium, alteration of $\mathrm{Ca}^{2+}$ handling and autonomic innervation (McMahon et al. 1996, 2002, Kennedy et al. 2003), show an evidence of systolic dysfunction in subtotally nephrectomized rats. Unfortunately, our experimental methods do not allow us to express an opinion on diastolic function. We can only speculate that the left ventricular diastolic function is also altered by decreased compliance of left myocardium due to considerable concentric hypertrophy. The left ventricular hypertrophy evident in our model by the relative increase in the left/right ventricle weight ratio is an alteration of multifactorial origin; hypertension, anemia, hyperparathyroidism, volume expansion and other risk factors have been implicated (Middleton et al. 2001).

The action potential duration determines the refractory period of cardiomyocyte and it is important for maintenance of normal regular heartbeat. Alteration of cardiac ionic currents leading to the shortening of action potential, pressure and volume overload, ventricular hypertrophy and dilation, electrolyte dysbalance and oxidative stress are the important proarrhythmogenic factors in CRF patients (Meier et al. 2001,
Korantzopoulos et al. 2007). Shortening of action potential in subtotally nephrectomized rats detected in our experiments at $50 \%$ and $90 \%$ levels of repolarization is probably caused by rapid inactivation of L-type calcium current (Donohoe et al. 2000a) and abnormalities of repolarizing potassium current (Donohoe et al. 2000b).

The surgical induction of experimental CRF and a quite fast onset of uraemia might diminish a direct clinical application of 5/6 nephrectomy. Nevertheless, the model closely resembles CRF in humans including cardiovascular complications, shows high degree of reproducibility and minimum of undesirable side-effects. Thus, the present rat model of subtotal surgical renal mass reduction represents a useful tool in the study of chronic renal failure and its cardiovascular complications.

\section{Conflict of Interest}

There is no conflict of interest.

\section{Acknowledgements}

The present study was supported by the Research project MSM 0021620819: Replacement of and Support to Some Vital Organs awarded by the Ministry of Education, Youth and Sports of the Czech Republic. We thank Renata Št'astná and Ivana Kušková for their excellent technical assistance.

\section{References}

BOUDET J, MAN NK, PILS P, SAUSSE A, FUNCK-BRENTANO JL: Experimental chronic renal failure in rat by electrocoagulation of renal cortex. Kidney Int 14: 82-86, 1978.

BRENNER BM, ANDERSON S: The interrelationships among filtration surface area, blood pressure, and chronic renal disease. J Cardiovasc Pharmacol 19: 1-7, 1992.

BURKE SW, SOLOMON AJ: Cardiac complications of end-stage renal disease. Adv Ren Replace Ther 7: 210-219, 2000.

CHOW KM, LIU ZC, CHANG TM: Animal remnant kidney model of chronic renal revisited. Hong Kong J Nephrol 5: 57-64, 2003.

DEBELLE FD, NORTIER JL, HUSSON CP, DE PREZ EG, VIENNE AR, ROMBAUT K, SALMON IJ, DESCHODT-LANCKMAN MM, VANHERWEGHEM JL: The renin-angiotensin system blockade does not prevent renal interstitial fibrosis induced by aristolochic acids. Kidney Int 66: 1815-1825, 2004.

DONOHOE P, MCMAHON AC, WALGAMA OV, BERTASO F, DOCKRELL ME, CRAMP HA, MULLEN AM, SHATTOCK MJ, HENDRY BM, JAMES AF: L-type calcium current of isolated rat cardiac myocytes in experimental uraemia. Nephrol Dial Transplant 15: 791-798, 2000.

DONOHOE P, HENDRY BM, WALGAMA OV, BERTASO F, HOPSTER DJ, SHATTOCK MJ, JAMES AF: An altered repolarizing potassium current in rat cardiac myocytes after subtotal nephrectomy. J Am Soc Nephrol 11: 1589-1599, 2000.

FUKUDA S, KOPPLE JD: Chronic uremia syndrome in dogs induced by uranyl nitrate. Nephron 25: 139-143, 1980. 
GIORDANO M, MANZELLA D, PAOLISSO G, CALIENDO A, VARRICCHIO M, GIORDANO C: Differences in heart rate variability parameters during the post-dialytic period in type II diabetic and non-diabetic ESRD patients. Nephrol Dial Transplant 16: 566-573, 2001.

GRIFFIN KA, PICKEN M, BIDANI AK: Method of renal mass reduction is a critical modulator of subsequent hypertension and glomerular injury. J Am Soc Nephrol 4: 2023-2031, 1994.

HEIDEMANN HT, HOFFMANN K, INSELMANN G: Long-term effects of acetazolamide and sodium chloride loading on cisplatin nephrotoxicity in the rat. Eur J Clin Invest 20: 214-218, 1990.

KENNEDY D, OMRAN E, PERIYASAMY SM, NADOOR J, PRIYADARSHI A, WILLEY JC, MALHOTRA D, XIE Z, SHAPIRO JI: Effect of chronic renal failure on cardiac contractile function, calcium cycling, and gene expression of proteins important for calcium homeostasis in the rat. J Am Soc Nephrol 14: 90-97, 2003.

KORANTZOPOULOS P, KOKKORIS S, LIU T, PROTOPSALTIS I, LI G, GOUDEVENOS JA: Atrial fibrillation in end-stage renal disease. Pacing Clin Electrophysiol 30: 1391-1397, 2007.

LIU ZC, CHOW KM, CHANG TMS: Evaluation of two protocols of uremic rat model: partial nephrectomy and infarction. Ren Fail 25: 935-943, 2003.

LUGON JR, WARRAK EA, LUGON AS, SALVADOR BA, NOBREGA ACL: Revisiting autonomic dysfunction in end-stage renal disease patients. Hemodial Int 7: 198-203, 2003.

McMAHON AC, VESCOVO G, DALla LIBERA L, WYNNE DG, FLUCK RJ, HARDING SE, RAINE AE: Contractile dysfunction of isolated ventricular myocytes in experimental uraemia. Exp Nephrol 4: 144-150, 1996.

MCMAHON AC, GREENWALD SE, DODD SM, HURST MJ, RAINE AE: Prolonged calcium transients and myocardial remodelling in early experimental uraemia. Nephrol Dial Transplant 17: 759-764, 2002.

MEIER P, VOGT P, BLANC E: Ventricular arrhythmias and sudden cardiac death in end-stage renal disease patients on chronic hemodialysis. Nephron 87: 199-214, 2001.

MIDDLETON RJ, PARFREY PS, FOLEY RN: Left ventricular hypertrophy in the renal patient. J Am Soc Nephrol 12: 1079-1084, 2001.

OKUDA S, OH Y, TSURUDA H, ONOYAMA K, FUJIMI S, FUJISHIMA M: Adriamycin-induced nephropathy as a model of chronic progressive glomerular disease. Kidney Int 29: 502-510, 1986.

SAHIN M, KAYATAS M, URUN Y, SENNAROGLU E, AKDUR S: Performing only one cardiovascular reflex test has a high positive predictive value for diagnosing autonomic neuropathy in patients with chronic renal failure on hemodialysis. Ren Fail 28: 383-387, 2006.

SAVICA V, MUSOLINO R, Di LEO R, SANTORO D, VITA G, BELLINGHIERI G: Autonomic dysfunction in uremia. Am J Kidney Dis 38: 118-121, 2001.

TESǍ̌ V: Epidemiology and classification of chronic kidney disease (in Czech). Urolog pro praxi 4: 180-181, 2006.

VITA G, MESSINA C, SAVICA V, BELLINGHIERI G: Uraemic autonomic neuropathy. J Auton Nerv Syst 30: 179-184, 1990.

WANIC-KOSSOWSKA M, LEHMANN P, CZEKALSKI S: Left ventricular systolic and diastolic dysfunction in patients with chronic renal failure treated with hemodialysis. Pol Arch Med Wewn 109: 365-373, 2003.

ZOCCALI C, CICCARELLI M, MAGGIORE Q: Defective reflex control of heart rate in dialysis patients: evidence for an afferent autonomic lesion. Clin Sci 63: 285-289, 1982.

ZOCCALI C, BENEDETTO FA, TRIPEPI G, MALLAMACI F: Cardiac consequences of hypertension in hemodialysis patients. Semin Dial 17: 299-303, 2004. 